\title{
SÍNTESE E CARACTERIZAÇÃO DE UM NOVO COMPOSTO OBTIDO PELA REAÇÃO ENTRE HIDRETO DE TRIFENILESTANHO E ÁCIDO ( \pm -MANDÉLICO E AVALIAÇÃO DE SEU POTENCIAL BIOCIDA SOBRE O FUNGO Fusarium oxysporum f. sp.cubense
}

\author{
Synthesis, characterization and evaluation of the biocide effect on the fungus \\ Fusarium oxysporum f. sp. cubense of a new compound obtained \\ by reaction of triphenyltin hydride and ( \pm )-mandelic acid
}

\author{
Roberto Santos Barbiéri', Elysio Prado de Lima ${ }^{2}$,Samuel Ferreira da Silva ${ }^{3}$,Allan Kardec Carlos Dias ${ }^{4}$, \\ Maria das Graças Cardoso ${ }^{5}$,Vilma Reis Terra ${ }^{6}$
}

\begin{abstract}
RESUMO
O presente artigo refere-se à síntese e caracterização de um novo composto organoestânico, pela reação de ácido ( \pm )mandélico e hidreto de trifenilestanho, em meio de acetonitrila e sob refluxo, $\left[\left(\mathrm{C}_{6} \mathrm{H}_{5}\right)_{2} \mathrm{SnMand}_{2}\right]\left\{\mathrm{Mand}=\mathrm{C} \mathrm{H}_{5} \mathrm{CH}(\mathrm{OH}) \mathrm{COO}\right]$, identificado por análise elementar de carbono e hidrogênio, espectroscopia no infravermelho e espectrometria de massa de alta resolução, para o qual foi proposta estrutura octaédrica com o grupo fenila em posição trans. Verificou-se que o composto apresenta ação biocida sobre o fungo Fusarium oxysporum f. sp. cubense, sendo mais efetivo que o ácido ( \pm )-mandélico livre. No entanto, a atividade biocida do composto foi menos intensa que a observada para cloreto de estanho hidratado, acetato de trifenilestanho e hidreto de trifenilestanho, empregados para fins de comparação. Nos testes de germinação de conídios e microconídios do mesmo fungo, na presença de $\left[\left(\mathrm{C}_{6} \mathrm{H}_{5}\right)_{2} \mathrm{SnMand}_{2}\right]$, os índices de germinação ficaram abaixo de $11 \%$.
\end{abstract}

Termos para indexação: Compostos organoestânicos, ácido ( \pm )-mandélico, efeito biocida, Fusarium, Fusarium oxysporum f. sp. cubense.

\begin{abstract}
The present paper refers to the synthesis and characterization of a new organotin compound that was obtained by reaction of ( \pm )-mandelic acid with triphenyltin hydride in acetonitrile medium under reflux. According to hydrogen and carbon elemental analysis, infrared spectroscopy and high resolution mass spectrometry the formula of such compounds is $\left(\mathrm{C}_{6} \mathrm{H}_{5}\right)_{2} \mathrm{SnMand}_{2}\left\{\mathrm{Mand}_{2}\right.$ $=\mathrm{C}_{6} \mathrm{H}_{5} \mathrm{CH}(\mathrm{OH}) \mathrm{COO}$. An octahedral complex, with the phenyl groups in trans position was proposed for its structure. It was observed that this compound was active against the fungus Fusarium oxysporum f. sp. cubense. The biocide effect was more intense than the one observed for $( \pm$-mandelic acid. However, it was less efficient than tin chloride hydrate, triphenyltin acetate and triphenyltin hydride. In germination assays with conides and microconides of the same fungus in the presence of $\left[\left(\mathrm{C}_{6} \mathrm{H}_{5}\right)_{2} \mathrm{SnMand}_{2}\right]$, the germination rates were below $11 \%$.
\end{abstract}

Index terms: Organotin compounds; ( \pm )-mandelic acid; biocide effect, Fusarium, Fusarium oxysporum f. sp. cubense.

(Recebido para publicação em 22 de maio de 2004 e aprovado em 8 de agosto de 2005)

\section{INTRODUÇÃO}

A química de compostos organoestânicos é conhecida há cerca de 150 anos (FRANKLAND, 1852, citado por NICHOLSON, 1989; LÖWING, 1852, citado por NICHOLSON, 1989) e, durante 75 anos, despertou apenas interesse acadêmico. A partir de 1925 , com a primeira patente para um composto organoestânico, a química desta classe de substâncias passou a ser alvo de atenção pelo variado campo de aplicações (LUIJTEN, 1972).

$\mathrm{Na}$ área tecnológica, compostos organoestânicos são usados como estabilizadores do PVC (cloreto de polivinila) e de outros polímeros vinílicos, diante da ação da luz e do calor, além de serem empregados em processos de tratamento de água e na preservação de produtos têxteis

\footnotetext{
Químico e Biólogo, Mestre e Doutor em Físico-Química - Professor do Programa de Mestrado em Biotecnologia da Universidade Vale do Rio Verde/UninCor Três Corações, MG - Diretor Acadêmico da Faculdade de Minas/FAMINAS - Muriaé, MG - barbieri@faminas.edu.br

Biólogo, estudante de Mestrado em Biotecnologia - Universidade Vale do Rio Verde/ UninCor - Três Corações, MG.

${ }^{3}$ Matemático e Mestre em Biotecnologia - Professor da Faculdade de Minas/FAMINAS - Muriaé, MG.

${ }^{4}$ Químico, Mestre em Agroquímica e Agrobioquímica - Doutor em Ciências dos Alimentos -Professor da Universidade Vale do Rio Verde/UninCor -

Três Corações, MG - dias@tricor.com.br

${ }^{5}$ Química, Mestre e Doutora em Bioquímica - Professora do Departamento de Química da Universidade Federal de Lavras/UFLA - Cx. P. 3037 37.200-000 - Lavras, MG.

${ }^{6}$ Química, Mestre em Química Analítica - Doutora em Química Inorgânica - Professora do Centro Federal de Tecnologia/CEFET - Uberaba, MG vilmaterra@yahoo.com.br
} 
e de madeira. Compostos organoestânicos podem atuar como catalisadores na produção de espumas poliuretânicas, em reações de esterificação ou de transesterificação, na produção de silicones, e na polimerização de olefinas (DAVIES, 1997; DAVIS \& SMITH, 1982; OMAE, 1989; POLLER, 1970).

A partir de 1950, investigações sistemáticas demonstraram a ação de compostos organoestânicos em relação a fungos e bactérias, organismos marinhos, vermes parasíticos, insetos e caracóis aquáticos, entre outros. Atualmente são estudados compostos fitossanitários (fungicidas, antibacterianos, anti-helmínticos, inseticidas, repelentes e biocidas em geral), com conseqüentes aplicações em agricultura, veterinária, farmácia e medicina (KUMARI et al., 1994; LUIJTEN, 1972).

No meio ambiente, compostos organoestânicos tendem a degradar-se em dióxido de estanho - $\mathrm{SnO}_{2}$ (OMAE, 1989). Se esta degradação ocorrer rapidamente, então o uso de pesticidas baseados em compostos organoestânicos torna-se mais interessante que outras substâncias, como os biocidas cúpricos, mercuriais ou fosfóricos. No entanto, em ambientes marinhos, tem sido observado o surgimento de órgãos sexuais masculinos em fêmeas de caramujos, fenômeno denominado de imposex ou pseudo-hermafroditismo, associado à absorção de compostos tributil- e trifenilestânicos (FERNANDES, 2004; FERNANDEZ et al., 2002).

No presente trabalho foi descrita a preparação e a caracterização de um novo composto, $\left[\left(\mathrm{C}_{6} \mathrm{H}_{5}\right)_{2} \mathrm{SnMand}_{2}\right]$ $\left\{\right.$ Mand $\left.=\mathrm{C}_{6} \mathrm{H}_{5} \mathrm{CH}(\mathrm{OH}) \mathrm{COO}\right\}$. Sua atividade biocida foi testada em cultura do fungo Fusarium oxysporum f. sp. cubense, a qual foi comparada com a atividade biocida do ácido ( \pm )-mandélico e hidreto de trifenilestanho, precursores do complexo, e com cloreto de estanho hidratado e acetato de trifenilestanho comercial (princípio ativo do herbicida Hogosuzu, produzido comercialmente pela Bayer do Brasil).

\section{MATERIAL E MÉTODOS}

\section{Reagentes, solventes e microrganismos}

Todos os reagentes e solventes, de grau analítico, empregados nos experimentos descritos neste trabalho foram utilizados sem purificação prévia, tendo as seguintes fórmulas e procedências: ácido ( \pm )-mandélico - $\mathrm{C}_{6} \mathrm{H}_{5} \mathrm{CH}(\mathrm{OH}) \mathrm{COOH}$, Vetec; acetonitrila - $\mathrm{CH}_{3} \mathrm{CN}$, Quimex; hidreto de trifenilestanho - $\left(\mathrm{C}_{6} \mathrm{H}_{5}\right)_{3} \mathrm{SnH}$, Aldrich; éter etílico - $\left(\mathrm{C}_{2} \mathrm{H}_{5}\right)_{2} \mathrm{O}$; cloreto de estanho hidratado $\mathrm{SnCl}_{2} \cdot 2 \mathrm{H}_{2} \mathrm{O}$, Merck; e acetato de trifenilestanho comercial - $\left(\mathrm{C}_{6} \mathrm{H}_{5}\right)_{3} \mathrm{SnOOCCH}_{3}$ (herbicida Hokkosuzu200), Hokko do Brasil.

As culturas do fungo Fusarium oxysporum f. sp. cubense utilizadas para os testes de avaliação dos compostos foram obtidas por meio de repicagens de culturas padrão do fungo, cedidas pelo Departamento de Fitopatologia da Universidade Federal de Lavras, Lavras-MG.

\section{Síntese e caracterização do composto}

$\mathrm{O}$ complexo $\left[\left(\mathrm{C}_{6} \mathrm{H}_{5}\right)_{2} \mathrm{SnMand}_{2}\right]$ [Mand = $\left.\mathrm{C}_{6} \mathrm{H}_{5} \mathrm{CH}(\mathrm{OH}) \mathrm{COO}\right]$ foi obtido segundo procedimento adaptado de Terra (1997) e Terra et al. (1998), descrito a seguir:

Em balão de fundo redondo de $50 \mathrm{~mL}$, dissolveu-se $0,270 \mathrm{~g}(1,78 \mathrm{mmol})$ de ácido $( \pm)$-mandélico, $\mathrm{C}_{6} \mathrm{H}_{5} \mathrm{CH}(\mathrm{OH}) \mathrm{COOH}$, em $20 \mathrm{~mL}$ de acetonitrila e acrescentou-se $0,30 \mathrm{~mL}(0,412 \mathrm{~g}, 1,17 \mathrm{mmol})$ de hidreto de trifenilestanho, $\left(\mathrm{C}_{6} \mathrm{H}_{5}\right)_{3} \mathrm{SnH}$. Por ser sensível ao ar, este foi retirado do frasco original com seringa e adicionado à mistura no balão, no menor espaço de tempo possível, com a ponta da agulha da seringa imersa na solução. Manteve-se o sistema sob agitação magnética e refluxo lento por 24 horas. Reduziu-se o volume da mistura até metade e deixou-se em repouso, sob refrigeração a $-18^{\circ} \mathrm{C}$, até a formação de precipitado com aparência cristalina. $\mathrm{O}$ sólido foi separado por filtração, lavado com três porções de $2 \mathrm{~mL}$ de éter etílico e seco em pistola de Abderhalden, a $100{ }^{\circ} \mathrm{C}$, durante 2 horas. Obteve-se $0,315 \mathrm{~g}$ do produto, correspondente a $54,8 \%$ de rendimento.

Para estabelecer o ponto de fusão, sem correção, foi utilizado o equipamento Bücchi 535. Os espectros de infravermelho foram obtidos usando a técnica de pastilhas com brometo de potássio, no espectrofotômetro Shimadzu FTIR-8201A com transformada de Fourier $\left(4600-400 \mathrm{~cm}^{-1}\right)$. Ambos foram determinados no Departamento de Química da Universidade Federal de Lavras, Lavras-MG.

As análises elementares de carbono e hidrogênio foram realizadas no equipamento analisador elementar Perkin Elmer modelo 2400, e os espectros de massa de alta resolução foram obtidos no espectrômetro VG Autospec Micromass, ambos do Instituto de Química da Universidade de Campinas, Campinas-SP.

\section{Determinação do potencial biocida}

O complexo organoestânico preparado, $\left[\left(\mathrm{C}_{6} \mathrm{H}_{5}\right)_{2} \mathrm{SnMand}_{2}\right]$, teve seu potencial biocida avaliado sobre culturas do fungo Fusarium oxysporum f. sp. cubense. O método utilizado foi o bioanalítico "in vitro", observando-se o crescimento do microrganismo com 
diferentes concentrações do composto obtido, tendo como controle cloreto de estanho hidratado, hidreto de trifenilestanho e acetato de trifenilestanho comercial.

Todos os testes foram conduzidos em meio de cultura BDA (batata, dextrose, ágar), acrescido de $20 \mathrm{~mL}$ do antibiótico Quemicetina/100 mL do meio, para evitar o desenvolvimento de bactérias. Para cada composto foram preparadas duas placas testemunhas, cada uma com 25 $\mathrm{mL}$ do meio de cultura e quatro placas de testes de crescimento micelial para cada uma das concentrações estudadas, 1, 10, 50, 100 e 500 ppm, exceto para o ácido ( \pm mandélico, que foi empregado nas concentrações de 100 e $500 \mathrm{ppm}$. Estas placas foram vedadas e levadas a uma câmara de incubação a $23-25^{\circ} \mathrm{C}$ durante 6-8 dias. Durante este período de crescimento, a cada 2-3 dias, foram feitas medições ortogonais do dos diâmetros das colônias com o uso de paquímetro, tendo como referência o crescimento da placa testemunha preenchida (DIAS, 1999; FERNANDEZ, 1993; SILVA, 2000).

Para avaliação de germinação de conídios e microconídios do Fusarium oxysporum f. sp. cubense foram preparadas duas placas testemunhas e quatro placas de testes do composto $\left[\left(\mathrm{C}_{6} \mathrm{H}_{5}\right)_{2} \mathrm{SnMand}_{2}\right]$. Cada placa de teste continha uma lâmina de vidro, sobre a qual colocouse $20 \mathrm{~mL}$ de solução do composto organoestânico na concentração de 500 ppm e $20 \mathrm{~mL}$ de solução micelial do Fusarium oxysporum. Depois de 10 horas em câmara a 18 ${ }^{\circ} \mathrm{C}$, adicionou-se $20 \mathrm{~mL}$ de solução do corante Lacto Fenol/ Azul de Tripan, levou-se ao microscópio, com aumento de 40x, para contagem de conídios e microconídios, germinados e não-germinados, em cada um dos quatro quadrantes das amostras nas lâminas, sendo os resultados expressos como médias aritméticas simples (DIAS, 1999; FERNANDEZ, 1993; SILVA, 2000).

\section{RESULTADOS E DISCUSSÃO}

O novo composto organoestânico $\left[\left(\mathrm{C}_{6} \mathrm{H}_{5}\right)_{2} \mathrm{SnMand}_{2}\right]$ obtido da reação de hidreto de trifenilestanho com o ácido ( \pm )-mandélico foi caracterizado mediante análise elementar de carbono e hidrogênio, espectroscopia vibracional no infravermelho e espectrometria de massa (Tabela 1).

O composto organoestânico obtido, de cor branca, quando observado ao microscópio, apresenta natureza microcristalina. Nas tentativas para recristalização do sólido obtido, não foi possível a obtenção de monocristais com características adequadas para determinação de estruturas por difratometria de raios-X.

Dos resultados de análise elementar de carbono e hidrogênio, pode-se estabelecer a fórmula molecular proposta para o composto, $\mathrm{C}_{28} \mathrm{H}_{24} \mathrm{O}_{6} \mathrm{Sn}$, cuja massa fórmula é concordante com o valor obtido por espectrometria de massa.

No espectro de infravermelho do novo composto organometálico, são de interesse as regiões de $3395-3400 \mathrm{~cm}^{-1}$ e $1060-1070 \mathrm{~cm}^{-1}$, relativas aos estiramentos da hidroxila alcoólica, e a região de 1560$1726 \mathrm{~cm}^{-1}$, relativa ao estiramento assimétrico da carboxila.

Da comparação do espectro de infravermelho do $\left[\left(\mathrm{C}_{6} \mathrm{H}_{5}\right)_{2}\right.$ SnMand $\left.{ }_{2}\right]$ com o espectro do ácido $( \pm)$ mandélico livre, pode-se verificar um deslocamento da banda $1716 \mathrm{~cm}^{-1}$, atribuída ao estiramento assimétrico da carboxila, „COO(assim) para $1560 \mathrm{~cm}^{-1}$ no complexo. Essas observações são consideradas evidências para a formação de complexos (BARBIÉRI et al., 1988, 1994, 1995, 2002; BOLARD, 1965; LARSEN \& HOMEIR, 1972).

TABELA 1 - Dados de ponto de fusão, análise elementar, espectrometria de massa e bandas significativas no espectro de infravermelho para $\left[\left(\mathrm{C}_{6} \mathrm{H}_{5}\right)_{2} \mathrm{SnMand}_{2}\right]$.

\begin{tabular}{|c|c|c|c|c|c|c|c|}
\hline \multirow{3}{*}{$\begin{array}{l}\text { Complexo } \\
\text { Fórmula }\end{array}$} & \multirow{3}{*}{$\begin{array}{l}\text { Ponto } \\
\text { de fusão } \\
\left({ }^{\circ} \mathbf{C}\right)\end{array}$} & \multirow{2}{*}{\multicolumn{2}{|c|}{$\begin{array}{c}\text { Análise Elementar } \\
\text { \% Experimental (\% } \\
\text { Teórica) }\end{array}$}} & \multirow{3}{*}{$\begin{array}{l}\text { Massa Fórmula } \\
\text { Experimental } \\
\text { (Teórica) }\end{array}$} & \multicolumn{3}{|c|}{ Infravermelho $\left(\mathrm{cm}^{-1}\right)$} \\
\hline & & & & & \multicolumn{2}{|c|}{ Ligante livre } & \multirow{2}{*}{$\begin{array}{l}\text { Complexo } \\
v_{\mathrm{COO}(\text { assim })}\end{array}$} \\
\hline & & $\% \mathrm{C}$ & $\% \mathrm{H}$ & & $v_{\mathrm{OH}}$ & $v_{\mathrm{COO}(\text { assim) }}$ & \\
\hline $\begin{array}{c}{\left[\left(\mathrm{C}_{6} \mathrm{H}_{5}\right)_{2} \mathrm{SnMand}_{2}\right]} \\
\mathrm{C}_{28} \mathrm{H}_{24} \mathrm{O}_{6} \mathrm{Sn}\end{array}$ & $173 d$ & $\begin{array}{c}58,33 \\
(58,78)\end{array}$ & $\begin{array}{c}4,11 \\
(4,20)\end{array}$ & $\begin{array}{c}574,06 \\
(575,21)\end{array}$ & $\begin{array}{l}3395 \\
1060\end{array}$ & 1716 & 1560 \\
\hline
\end{tabular}

Mand $=\mathrm{C}_{6} \mathrm{H}_{5} \mathrm{CH}(\mathrm{OH}) \mathrm{COO} ; \mathrm{d}=$ decomposição 
Também se observa no espectro do composto organometálico, em relação ao respectivo espectro do ácido ( \pm )-mandélico livre, um significativo alargamento da banda cerca de $3400 \mathrm{~cm}^{-1}$, atribuída à hidroxila alcóolica no ácido, o que evidencia sua participação na coordenação ao estanho (HAMILTON \& IBERS, 1968; LARSEN \& HOMEIR, 1972; TERRA, 1991). Observa-se ainda no espectro do composto, em relação ao do ácido livre, alterações nas bandas na região $1050-1100 \mathrm{~cm}^{-1}$, também indicativas da participação da hidroxila alcoólica na coordenação ao estanho (BARBIÉRI, 1978; LARSEN \& HOMEIR, 1972).

O composto $\left[\left(\mathrm{C}_{6} \mathrm{H}_{5}\right)_{2} \mathrm{SnMand}_{2}\right]$ deve ser espécie monomérica, o que pode ser evidenciado também de seu espectro de infravermelho, em comparação com o do ácido livre. No espectro do ácido ( \pm )-mandélico podem ser percebidas bandas pouco intensas nas regiões aproximadamente em 1900 e $2600-2700 \mathrm{~cm}^{-1}$, atribuídas a ligações de hidrogênio intermoleculares nos ácidos livres, inexistentes no espectro do composto organoestânico derivado. Bandas nas mesmas regiões foram observadas em complexos de zircônio e háfnio, derivados de diversos ácidos a-hidroxicarboxílicos, indicativas de estruturas diméricas para estes compostos, estabilizados por ligações de hidrogênio intramoleculares (BARBIÉRI et al., 1988; LARSEN \& HOMEIR, 1972).

Há que destacar ainda o fato do novo complexo obtido ser de natureza diorganoestânica derivado de precursor triorganoestânico.

De modo geral, haletos organoestânicos reagem com ácidos carboxílicos, em presença de bases, como trietilamina, que atuam como agentes facilitadores da substituição do halogênio coordenado, pela formação de haleto de hidrogênio (NEUMAM, 1970; OMAE, 1989), que é eliminado na forma de cloridrato de trietilamina (KERK \& LUITJTEN, 1956), de acordo com a equação:

$$
2 \mathrm{R}_{3} \mathrm{SnX}+2 \mathrm{R}^{\prime} \mathrm{COOH} \underset{-2 \mathrm{HX}}{\stackrel{\mathrm{Et}_{3} \mathrm{~N}}{\longrightarrow}}\left[\mathrm{R}_{2} \mathrm{Sn}\left(\mathrm{OCOR}^{\prime}\right)_{2}\right]
$$

Quando a reação ocorre entre um sal de ácido carboxílico, a presença da base é dispensável (NEUMAM, 1970; OMAE, 1989):

$$
2 \mathrm{R}_{3} \mathrm{SnX}+\left(\mathrm{R}^{\prime} \mathrm{COO}\right)_{2} \mathrm{~Pb} \longrightarrow 2\left[\mathrm{R}_{3} \mathrm{SnOCOR}^{\prime}\right]+\mathrm{PbX}_{2}
$$

No entanto, pode haver reação entre ácidos carboxílicos e haletos organoestânicos, na ausência de base. Neste caso, ocorre a formação de carboxilatos haloestânicos, pela substituição de um grupo alquila por um grupo carboxilato:

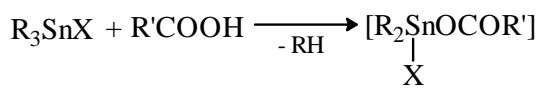

Na reação de hidreto de trifenilestanho com ácido ( \pm -mandélico, em meio de acetonitrila, foi observada a formação do novo composto $\left[\left(\mathrm{C}_{6} \mathrm{H}_{5}\right)_{2}\right.$ SnMand $\left._{2}\right]$, pela substituição do íon hidreto e de um radical fenila. $\mathrm{O}$ menor caráter básico da acetonitrila, em relação à trietilamina (DEAN, 1987), deve ter possibilitado uma condição intermediária de basicidade, que permitiu esta dupla substituição simultânea.

$$
\begin{gathered}
\mathrm{R}_{3} \mathrm{SnX}+\mathrm{C}_{6} \mathrm{H}_{5} \mathrm{CH}(\mathrm{OH}) \mathrm{COOH} \underset{ }{\stackrel{-\mathrm{R}}{\longrightarrow}}\left[\mathrm{R}_{2} \mathrm{SnL}_{2}\right] \\
-\mathrm{H}_{2} \\
\left\{\mathrm{R}=\mathrm{Ph} ; \mathrm{L}=\mathrm{C}_{6} \mathrm{H}_{5} \mathrm{CH}(\mathrm{OH}) \mathrm{COO}\right\}
\end{gathered}
$$

Com base nas informações obtidas foi possível propor a fórmula estrutural indicada na Figura 1 para o novo composto obtido.

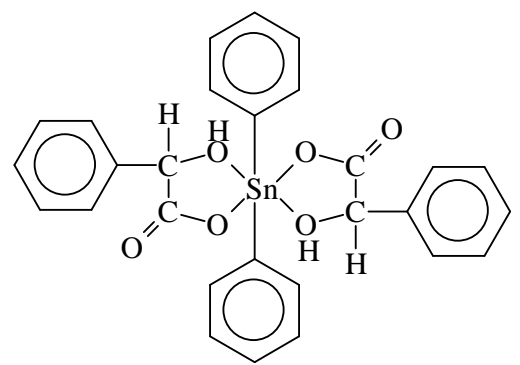

FIGURA 1 - Fórmula estrutural proposta para o composto $\left[\left(\mathrm{C}_{6} \mathrm{H}_{5}\right)_{2} \mathrm{SnMand}_{2}\right]$

Apesar do precursor da síntese, o hidreto de trifenilestanho, ser um composto sensível ao ar, o novo composto organometálico preparado mostrou-se estável, podendo ser exposto ao ar, sem necessidade de uso de técnicas de manipulação sob atmosfera inerte.

Para avaliar o efeito biocida, o novo composto foi aplicado em culturas do fungo Fusarium oxysporum $\mathrm{f}$. sp. cubense, nas concentrações de 1, 10, 50, 100 e 500 ppm. Para efeito de comparação, foram feitas aplicações de cloreto de estanho (II) hidratado, que é um composto inorgânico facilmente disponível, de acetato de trifenilestanho comercial (na forma do herbicida Hokkosuzu-200, fabricado pela Hokko do Brasil), de ácido $( \pm)$-mandélico e de hidreto de trifenilestanho. Os resultados obtidos estão indicados na Tabela 2. 
Vale salientar que, quando da aplicação de hidreto de trifenilestanho nas culturas de fungo, deve-se considerar que o mesmo é sensível ao ar e deve transformar-se em hidróxido de trifenilestanho e/ou óxido de bis(trifenilestanho) (DAVIES, 1997).

Os compostos estudados apresentaram um efeito inibitório do crescimento médio micelial do fungo Fusarium oxysporum f. sp. cubense mais acentuado, para todas as concentrações, em relação à placa testemunha, no entanto, em nenhuma das concentrações estudadas o efeito inibitório foi total.
Praticamente, o efeito inibitório do $\left[\left(\mathrm{C}_{6} \mathrm{H}_{5}\right)_{2} \mathrm{SnMand}_{2}\right]$ sobre o crescimento médio micelial do fungo foi maior que $\mathrm{o}$ observado para o ácido ( \pm )-mandélico livre sendo, porém, menor que os efeitos observados para cloreto de estanho hidratado, hidreto de trifenilestanho e acetato de trifenilestanho comercial.

$\left[\left(\mathrm{C}_{6} \mathrm{H}_{5}\right)_{2} \mathrm{SnMand}_{2}\right]$, na concentração de $1 \mathrm{ppm}$, foi utilizado também para avaliar os possíveis efeitos inibitórios na germinação de conídios e microconídios para Fusarium oxysporum f. sp. cubense. Os resultados obtidos estão indicados na Tabela $3 \mathrm{e}$, em todos os testes realizados, os valores dos índices de germinação ficaram abaixo de $11 \%$.

TABELA 2 - Crescimento médio micelial de Fusarium oxysporum f. sp. cubense em meio BDA na presença de cloreto de estanho hidratado, acetato de trifenilestanho comercial (herbicida Hokkosuzu-200), ácido ()-mandélico, hidreto de trifenilestanho e $\left[\left(\mathrm{C}_{6} \mathrm{H}_{5}\right)_{2} \mathrm{SnMand}_{2}\right]$.

\begin{tabular}{lcc}
\hline \multicolumn{1}{c}{ Substância } & $\begin{array}{c}\text { Concentração } \\
(\mathbf{p p m})\end{array}$ & $\begin{array}{c}\text { Crescimento médio micelial } \\
(\mathbf{c m})\end{array}$ \\
\hline Cloreto de estanho hidratado & 1 & 0,28 \\
& 10 & 0,34 \\
& 50 & 0,29 \\
& 100 & 0,21 \\
& 500 & 0,12 \\
\hline Acetato de trifenilestanho comercial (herbicida & 1 & 0,08 \\
Hokkosuzu-200) & 10 & 0,09 \\
& 50 & 0,06 \\
& 100 & 0,03 \\
& 500 & 0,03 \\
\hline Ácido $( \pm)$-mandélico & 100 & 0,50 \\
& 500 & 0,33 \\
\hline Hidreto de trifenilestanho & 1 & 0,09 \\
& 10 & 0,30 \\
& 50 & 0,09 \\
& 100 & 0,06 \\
& 500 & 0,05 \\
\hline$\left(\mathrm{C}_{6} \mathrm{H}_{5}\right)_{2}$ SnMand & & 0,27 \\
& 1 & 0,26 \\
& 10 & 0,28 \\
& 50 & 0,27 \\
\hline Placa testemunha & 100 & 0,21 \\
\hline
\end{tabular}

TABELA 3 - Testes de germinação de conídios e microconídios para Fusarium oxysporum $\mathrm{f}$. sp. cubense na presença de $\left[\left(\mathrm{C}_{6} \mathrm{H}_{5}\right)_{2} \mathrm{SnMand}_{2}\right]$ na concentração de $500 \mathrm{ppm}$.

\begin{tabular}{ccccc}
\hline Lâmina & \multicolumn{2}{c}{ Médias das Contagens } & \multicolumn{2}{c}{ Índice de Germinação (\%) } \\
\hline & Germinação & Não-germinação & Germinação & Não-germinação \\
\hline 1 & 4,0 & 33,0 & 10,8 & 89,2 \\
3 & 1,0 & 20,0 & 4,8 & 95,2 \\
4 & 7,5 & 103,5 & 6,8 & 93,2 \\
\hline
\end{tabular}




\section{CONCLUSÕES}

Os resultados obtidos demonstraram que o novo composto organoestânico preparado, $\left[\left(\mathrm{C}_{6} \mathrm{H}_{5}\right)_{2} \mathrm{SnMand}_{2}\right]$, apresenta efeito inibitório no crescimento médio micelial de Fusarium oxysporum f. sp. cubense em maior intensidade que o ácido ( \pm )-mandélico livre, sendo menos eficiente que cloreto de estanho hidratado, acetato de estanho comercial e hidreto de trifenilestanho.

Também se destaca que o composto $\left[\left(\mathrm{C}_{6} \mathrm{H}_{5}\right)_{2} \mathrm{SnMand}_{2}\right]$, mesmo apresentando menor efeito biocida relativo a seu precursor, o hidreto de trifenilestanho, apresenta como principais vantagens os fatos de ser estável ao ar e inodoro, em contraste com o forte, irritante, penetrante e tóxico odor do hidreto de trifenilestanho usado na síntese do composto, o que o torna plausível de utilização, por exemplo, na sanitização de materiais ou ambientes usados ou freqüentados por humanos.

\section{REFERÊNCIAS BIBLIOGRÁFICAS}

BARBIÉRI, R. S. Estudo por correlação angular perturbada diferencial de alguns glicolatos de háfnio(IV). 1978. $66 \mathrm{f}$. Dissertação (Mestrado em Físico-Química) - Universidade Federal de Minas Gerais, Belo Horizonte, 1978.

BARBIÉRI, R. S.; DIAS, A. K. C.; CARDOSO, M. das G. Thermal analyses of diorganotin $d l$-mandelates. Journal of Thermal Analysis and Calorimetry, Budapeste, v. 67, p. 453-458, 2002.

BARBIERI, R. S.; KLEIN, S. I.; MARQUES NETTO, A. Time differential perturbed angular correlation (TDPAC) study some hafnium glycolates complexes. Polyhedron, London, v. 7, n. 21, p. 2165-2169, July 1988.

BARBIÉRI, R. S.; TERRA, V. R.; MARQUES NETTO, A.; ROCHA, J. C. Infrared study of zirconium and hafnium ahydroxycarboxylates. Eclética Química, São Paulo, v. 19, p. 129-135, 1994.

BARBIÉRI, R. S.; TERRA, V. R.; MARQUES NETTO, A.; ROCHA, J. C. Contribution to the study of zirconium and hafnium a-hydroxycarboxylates. Química Nova, São Paulo, v. 18, n. 1, p. 40-43, jan./fev. 1995.

BOLARD, J. J. Infrared spectra of tartaric acid and simple tartrates. Journal de Chemie Physique, Paris, v. 62, n. 7-8, p. 894-899, juil./août. 1965.
DAVIES, A. G.; SMITH, P. J. Tin. In: WILKINSON, G.; STONE, F. G. A.; ABEL, E. W. Comprehensive organometallic chemistry: the syntheses, reactions and structures of organometallic compounds. Oxford: Pergamon, 1982.835 p.

DAVIES, Alwyn G. Organotin chemistry. New York: Basel, 1997. $328 \mathrm{p}$.

DIAS, A. K. C. Efeito biocida de alguns ácidos ahidroxicarboxílicos e compostos organoestânicos sobre Fusarium oxysporum f. sp. cubense e Phytophthora capsici. 1999. 54 p. Dissertação (Mestrado em Agroquímica e Agrobioquímica) - Universidade Federal de Lavras, Lavras, 1999.

DEAN, J. A. Handbook of organic chemistry. New York: McGraw Hill, 1987. Não paginado.

FERNANDES, T. Transformação sexual no mar. Ciência Hoje, Rio de Janeiro, v. 35, n. 207, p. 34-35, ago. 2004.

FERNANDEZ, M. A. et al. Occurrence of imposex in Thais haemastoma: possible evidence of environmental contamination derived from organotin compounds in Rio de Janeiro and Fortaleza, Brazil. Cadernos de Saúde Pública, Rio de Janeiro, v. 18, n. 2, p. 463-476, mar./abr. 2002.

FERNANDEZ, M. R. Manual para laboratório de fitopatologia. Passo Fundo: EMBRAPA-CNTP, 1993.

HAMILTON, W. C.; IBERS, J. A. Hydrogen bonding in solids. New York: Benjamin, 1968. 284 p.

KERK, G. J. M. van der; LUIJTEN, J. G. A. Investigations on organotin compounds: IV. the preparation of a number of trialkyl and triaryl compounds. Journal of Applied Chemistry, London, v. 6, p. 49-55, 1956.

KUMARI, A.; SINGH, I.; TANDON, J. P. Coordination behavior and microbial studies of organotin(IV) complexes of biologically active heterocyclic benzothiazolines. Main Group Metal Chemistry, Tel Aviv, v. 17, n. 5, p. 347-361, may 1994.

LARSEN, E. M.; HOMEIR, E. H. Zirconium(IV) and hafnium(I) complexes of a-hydroxycarboxylates, lactates, mandelates and isopropylmandelates: stereospecificity in eight-coordinate complexes. Inorganic Chemistry, Washington, v. 11, n. 11, p. 2687-2692, Nov. 1972. 
LUIJTEN, J. G. A. Applications and biologicals effects of organotin compounds. In: SAWYER, A. Organotin compounds. New York: M. Dekker, 1972. v. 3, p. 921-974.

NEUMAM, W. P. The organic chemistry of tin. London: J. Wiley, 1970. 232 p.

NICHOLSON, J. W. The early history of organotin chemistry. Journal of Chemical Education, Washington, v. 66, n. 8, p. 621-622, Aug. 1989.

OMAE, I. Organotin chemistry. Amsterdam: Elsevier, 1989. $320 \mathrm{p}$.

POLLER, R. C. The chemistry of organotin compounds. London: Logos, 1970. 315 p.

SILVA, R. A. Avaliação de extratos vegetais na inibição "in vitro" de fungos patogênicos. 2000. 47 f. Dissertação
(Mestrado em Agroquímica e Agrobioquímica) Universidade Federal de Lavras, Lavras, 2000.

TERRA, V. R. Compostos organoestânicos com ácidos ahidroxicarboxílicos e a-aminoácidos. 1997. 109 f. Tese (Doutorado em Química Inorgânica) - Universidade Federal de Minas Gerais, Belo Horizonte, 1997.

TERRA, V. R. Estudo analítico de a-hidroxicarboxilatos de zircônio e de háfnio. 1991. 120 f. Dissertação (Mestrado em Química Analítica) - Universidade Estadual Paulista “Júlio de Mesquita Filho", Araraquara, 1991.

TERRA, V. R.; BARBIÉRI, R. S.; CASTELO BRANCO, P. de A.; ABRAS, A. Síntese e caracterização de compostos diorganoestânicos com ácido $d l$ mandélico. Eclética Química, São Paulo, v. 23, p. 1730, 1998. 